\section{Application of the Gatan X-ray Ultramicroscope (XuM) \\ to the Investigation of Material and Biological Samples}

\author{
Paul Mainwaring \\ Gatan, Inc., Pleasanton, CA \\ pmainwaring@gatan.com
}

\section{Introduction}

$\mathrm{X}$-ray ultramicroscopy in the SEM is a relatively new application in the wider field of X-ray microscopy. This latter field includes synchrotron and cabinet-based systems that vary in their X-ray power, capability, sample size, spatial resolution, and convenience. One important capability of the SEM-hosted X-ray microscope is that the normal SEM imaging and analytical functions such as secondary and backscattered imaging and microanalysis by EDX or WDS are unimpeded. X-ray imaging then serves as a complement to the normal use of the SEM. The convenience of easy access in an SEM lab to an X-ray microscope with 3D tomographic capability makes this an important development.

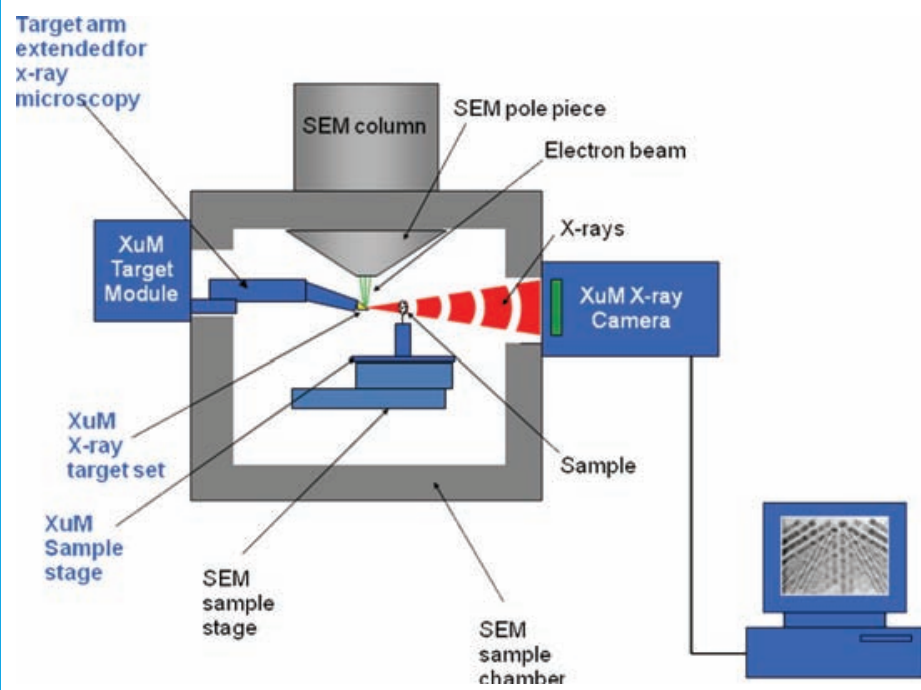

Fig 1: Schematic illustration showing XuM in operational configuration.

Target arm

retracted for

normal SEMuse

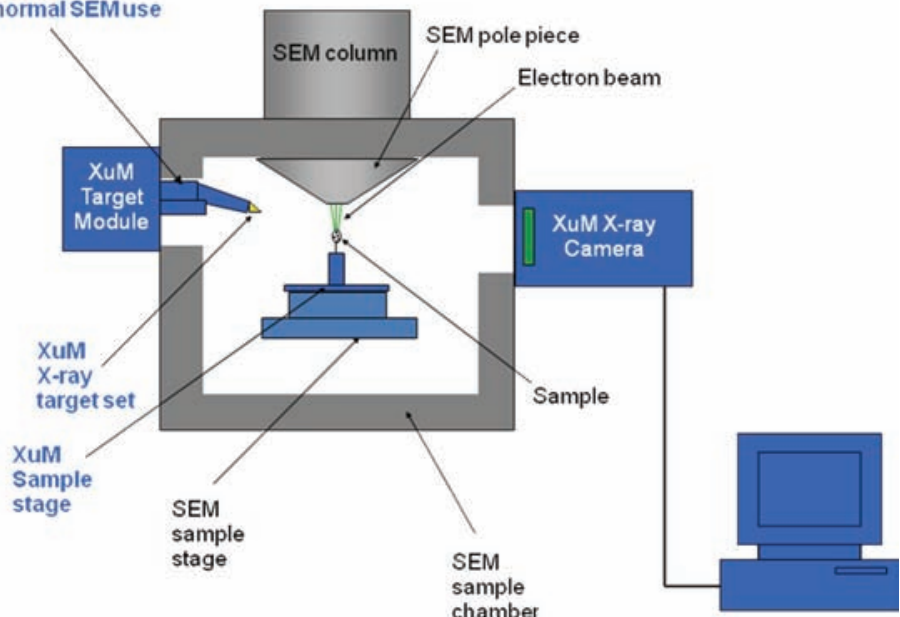

Fig 2: Schematic illustration showing normal SEM mode. Note that there is no conflict with normal SEM usage.
The Gatan X-ray Ultramicroscope or XuM, is a high $\mathrm{p}$

SEM-hosted X-ray Microscope (XRM) that enables non-destructive imaging of the internal structure of samples. Both two-dimensional (2D) X-ray imaging and three-dimensional tomographic imaging and reconstructions are possible with the XuM. The system is interfaced to and integrated with the SEM host to provide an easy to use, powerful $\mathrm{x}$-ray generation facility with unique imaging capability suitable for both life and materials science applications.

\section{Design of the X-ray Microscope System}

A schematic illustration of the components of the XuM is shown in Fig. 1. The SEM electron beam is focused onto a target to generate a point-like X-ray source. The generated X-rays are transmitted through the sample and projected onto a sensitive X-ray camera to form an image. This 'point-projection geometry' means that the sample is always in focus and magnification changes involve only moving the sample towards and away from the point source in front of the CCD camera. The target-carrying arm can be retracted to enable regular SEM work including stage tilting, as shown in Fig. 2. XuM and SEM can therefore be used as complimentary techniques-XuM to image the internal structures as well as external features of the sample and the SEM to image the external surface in high resolution. Thus, the XuM rather simply adds a third dimension to the SEM capability.

\section{XuM Imaging of 2 Samples \\ Geological Particles}

An example of the combined use of the XuM and electron imaging in the SEM is that of a lunar dust particle surrogate. The XuM and electron images of the particle are shown in Fig. $3 \mathrm{a}$ and Fig. 3b respectively. The electron image, shows the surface features of the particle clearly, but offers no indication of the presence of vesicles and voids present beneath the surface. The XuM image not only reveals these internal features, but also offers valuable information about their shape, size, and distribution density.
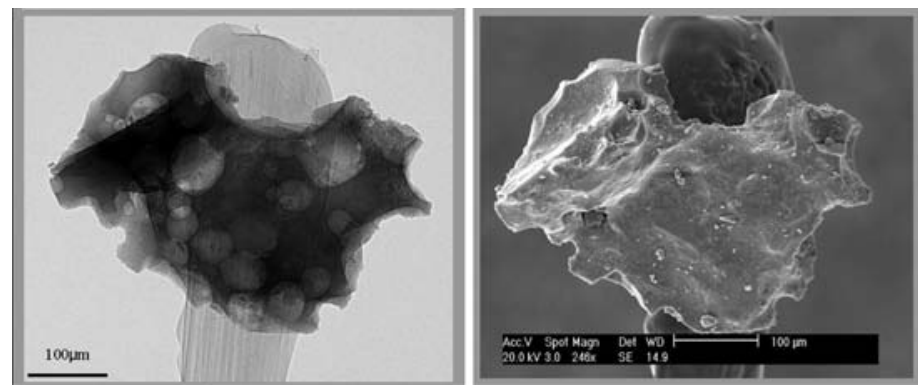

Fig 3a (left): XuM image of a lunar dust particle surrogate reveals internal features not visible on the surface.

Fig $3 b$ (right): SEM image of the same lunar dust particle surrogate showing only surface features.

It should be noted that traditional X-ray imaging techniques employ only absorption contrast as the mechanism of image formation. Absorption contrast depends on both the energy of the incident x-ray beam, the attenuating properties of structures within the sample, and the thickness of those structures. The logarithm of the transmitted $\mathrm{X}$-ray intensity is proportional to the projected density of the object. Absorption will thus be greater for greater densities or total thickness of the sample. Absorption-contrast X-ray images provide information about differences in the attenuating (absorbing) properties and the thickness of structures within the sample.

The XuM takes advantage of not only the absorption contrast mechanism but also a phase contrast mechanism, which exploits the wave properties of X-rays. Phase contrast arises from the refraction 
Frozen hydrated microtubules prepared using Cryoplunge ${ }^{T M} 3$. Image was recorded at TEM magnification of $59 \mathrm{kX}$ and electron dose of $20 \mathrm{e}-/$ $\AA^{2}$ at $300 \mathrm{keV}$ using Gatan Ultrascan ${ }^{\mathrm{TM}}$ 4000 . Image courtesy of Dr. Chen $\mathrm{Xu}$, Rosenstiel Basic Medical Sciences Research Center, Brandeis University, Waltham, MA.

WWW.goton.com/onswers

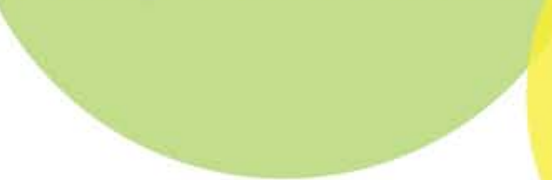

frozen hydrated microtubules 


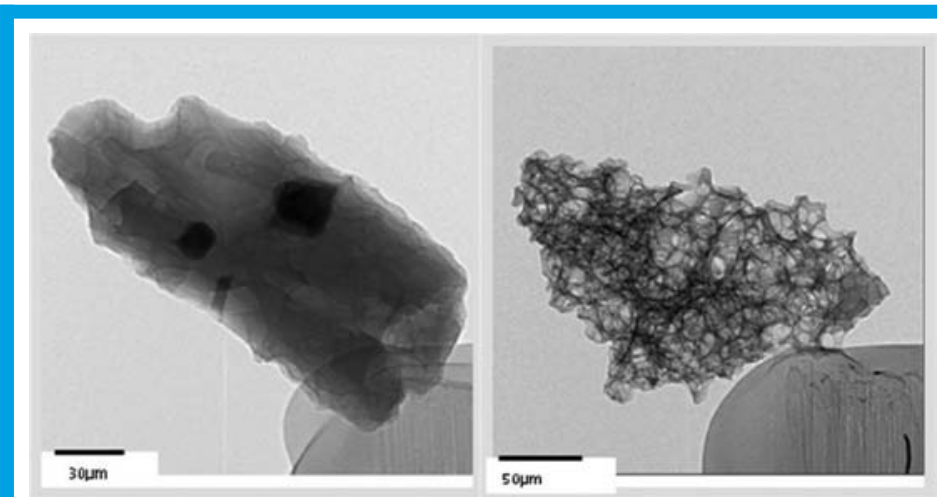

Fig 4a (left): XuM image of a volcanic basalt particle revealing features enhanced primarily by absorption contrast.

Fig $4 b$ (right): XuM image of a volcanic ash particle revealing features significantly enhanced by phase contrast.

(rather than absorption) of $\mathrm{x}$-rays by the sample, and is therefore sensitive to changes in refractive index. Large variations in refractive index can occur in samples that present very little variation in density (and hence show little absorption contrast) and phase-contrast imaging can be employed to image these otherwise invisible details. The phase of $\mathrm{x}$-rays passing through the sample is differentially sensitive to bulk and interfaces even though no mass difference is present. When the wave is allowed to propagate far enough beyond the sample, Fresnel diffraction will occur. This interference results in an intensity change visible as characteristic black/white fringes at the edges of an object in the image, leading to edge enhancement. Samples that show significant absorption contrast can also benefit by edge enhancement.

An example that shows both phase features and absorption features involves volcanic ash particles from the Hayes volcano in Alaska. The morphologies of the ash particles were investigated by C. Kiely


Fig. 5 a (upper left): XuM tomogram (inverted contrast) of a bryozoan sample prior to phase retrieval.

Fig. $5 b$ (upper right): Intensity profile across the edge indicated in Fig. $5 a$.

Fig. $6 a$ (bottom left): XuM tomogram (inverted contrast) of a bryozoan sample after phase retrieval. Fig. $6 a$.

Fig. $6 b$ (bottom right): Intensity profile across the edge indicated in

mICROSCOPY TODAY November 2008 et al. of Lehigh University with an XuM to gain mechanistic insights into the various magma processes that occur prior to, during, and after eruption. Fig. 4a is an XuM image of a volcanic basalt particle. Three inclusions of more dense minerals occur as mainly absorption features in the particle; these most likely correspond to iron oxide in composition and are clearly observed inside the particle. Fig $4 \mathrm{~b}$ is an XuM image of another ash particle that reveals many overlapping spherical gas bubbles trapped in various stages of coalescence. Phase contrast is responsible for boundary enhancement and can be seen in the black/white fringes (Fresnel) around the voids.

Fresnel fringes are however, undesirable for accurate 3D reconstruction of tomography data sets. It is important to remove them from the edges/interfaces prior to reconstruction since they can lead to creation of artifacts in the final reconstruction.

\section{Bryozoan Fossil - Tomographic Reconstruction}

Bryozoans are small, colonial animals that generally build stony skeletons of calcium carbonate. The bryozoans' skeletons grow in a variety of shapes and patterns: mound-shaped, lacy fans, branching twigs, and even corkscrew-shaped. Their skeletons have numerous tiny openings, each of which is the home of a minute animal called a zooid. There are about 6,000 living species of bryozoans and several times that number of fossil species. Bryozoans have a rich but insufficiently investigated fossil history. Scientists in the Department of Paleontology at the Natural History Museum, London, who have contributed significantly to the understanding of bryozoan fossils through their SEM investigations of bryozoan skeletal ultrastructures and the zooidal microstructures, desired a more powerful visualization tool-one that would allow them to obtain a 3D rotatable model of the Bryozoan sample and permit sectioning in arbitrary ways to reveal internal features. Specific objectives in this case were to study the size, structure and formation of branches and the tiny openings that are home to zooids.

The Gatan XuM was used to acquire many 2D projections of bryozoans mounted on a rotating tomography sample stage in the SEM using steps of 0.5 degrees (720 images in total). As mentioned earlier, phase contrast was removed from the tomograms using phase retrieval algorithms in order to eliminate artifacts during reconstruction. An inverted contrast tomogram of the bryozoan sample before and after phase retrieval is shown in Fig. 5a and Fig. 6a respectively. The corresponding intensity profiles at the indicated edge before and after phase retrieval are shown in Fig. 5b and Fig 6b respectively. Phase contrast manifests as a spike in intensity across the edge of the
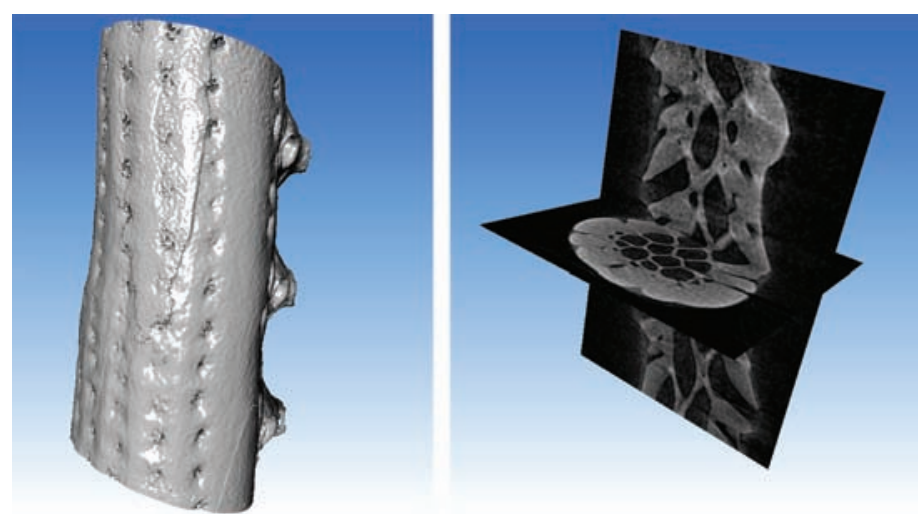

Fig. 7 a (left): 3D reconstruction of a bryozoan fossil sample showing external features.

Fig. $7 b$ (right): Ortho-slices through the bryozoan sample showing internal features. 


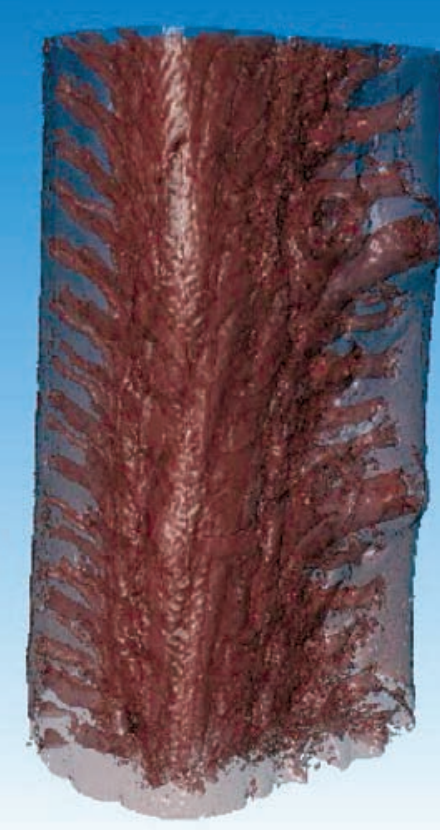

Fig. 8: The reconstructed image of a semi-transparent isosurface of the bryozoan fossil sample.

sample, as seen in Fig. 5b, in the pre-phase retrieved image. After phase retrieval, a flattening of the intensity spike occurs, as seen in Fig. 6b. The phase-retrieved tomograms were subsequently used to reconstruct a successful 3D model of the Bryozoan sample. The 3D model revealed the outer skeletal structure of the branches and the numerous tiny openings for the zooid, as observed in Fig. 7a. In addi- tion, ortho-slices (i.e. slices taken in the orthogonal direction) through the model distinctly revealed the internal structures, as seen in Fig 7b, that lead to the branches and the openings, thereby providing vital clues about their formation.

Figure 8 shows the reconstructed image of a semi-transparent isosurface of the bryozoan fossil sample. The internal branching structure of the bryozoan fossil sample is clearly seen. Using the visualization tools in the software various projections and 'fly-throughs' can be produced.

\section{Summary}

Gatan's X-ray microscope XuM and the host SEM were used as complimentary tools to image both internal and external features of the lunar dust particle surrogate. Fine internal details of volcanic ash particles from the Hayes volcano in Alaska were successfully imaged by taking advantage of the XuM's ability to employ both absorption contrast and phase contrast mechanisms that are available in the X-ray image. Rotation series of the bryozoan fossil sample were acquired, phase retrieved, and reconstructed to successfully create high-quality 3D tomogram that was "electronically sectioned" to reveal intricate internal structures. The ease of use and convenience of having such an $\mathrm{X}$-ray microscope in an SEM laboratory should lead to more thorough investigations of similar samples in the near future.

\section{Acknowledgements:}

1. Lunar dust surrogate sample and images courtesy of C. Kiely and C. J. Kiely, Lehigh University, Bethlehem, PA.

2. Volcanic ash samples and images courtesy of C. Kiely, D. Sahagian and C. J. Kiely, Lehigh University, Bethlehem, PA.

3. Bryozoan fossil sample courtesy of A. Ball and L. Howard of the Natural History Museum, London.

4. J .A. Hunt and B. C. Gundrum, Gatan Inc., Pleasanton, CA.

\section{STOP HASSLING WTH \\ MULTIPLE SERVICE CONTRACTS!}

START by putting all of our instruments under one service contract with MAS (regardless of make or model). Our expert EM SERVICE GROUP has the knowledge and skills to keep your instrument working at its best.

\section{TEM'S / SEM'S PREP EQUIPMENT SPECIAL SERVICES}

\begin{tabular}{cc|c} 
HITACHI & TOPCON & VACUUM COATERS \\
JOEL & ISI & PLASMA ASHERS \\
AMRAY & CAMBRIDGE & SPUTTER COATERS \\
& & ION MILLS
\end{tabular}

STAGES

\section{BEAM BLANKERS}

CUSTOM DEVICES

\section{NEW NEW NEW}

\section{Authorized service representatives}

for Gatan preparation equipment and Topcom TEM'S.
Contracts and On-Demand Emergency Service at

Reasonable Rates from

Factory Trained Specialist

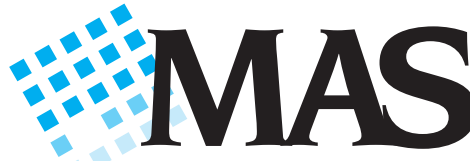

\section{0-421-8451}

3945 Lakefield Court Suwanee, Georgia 30024 770-866-3200 FAX 770-866-3259 ADVANGED ANALYTICAL PRODUCTS AND SERVICES 